\title{
A New Early Termination Scheme of Iterative Turbo Decoding Using Decoding Threshold
}

\author{
Fan-Min Li, Cheng-Hung Lin, and An-Yeu (Andy) Wu \\ Graduate Institute of Electronics Engineering, and Department of Electrical Engineering, \\ National Taiwan University
}

\begin{abstract}
Although many stopping methods of iterative decoding have been discussed in the literatures extensively, many of them only focus on the solvable decoding. In this paper, we propose a new early termination (ET) scheme based on the decoding threshold. Moreover, we proposed the cross-correlation measurement to predict the decoding threshold. The iterative decoding can stop in either high-SNR situation where the decoded bits are highly reliable (solvable decoding), or in low-SNR situation where the decoder already has no capability to decode (unsolvable decoding). At the same time, the receiver can send an automatic-repeat-request (ARQ) message to the transmitter in noise-seriously-coupling situation. Through simulations, the reduced iterations due to the ET scheme almost will not affect the SNR performance. Based on our analysis and simulation results, we can further modify the conventional GENIE chart. The ET property can help to either reduce the computational complexity or power consumptions in implementing the iterative decoding in DSP processors or VLSIs in mobile handsets.
\end{abstract}

\section{INTRODUCTION}

In 1993, a new class of Forward-Error-Correction (FEC) code, Turbo code, was introduced by Berrou, Glavieux and Thitimajashima [1][2]. The basic concept of this new decoding scheme is to use a parallel concatenation of at least two codes with an interleaver between the encoders. Decoding is based on alternately decoding the component codes, and passing the so-called extrinsic information (a part of the soft output of the soft-in/soft-out decoder) to the next decoding stage. The Turbo code is well known for its near Shannon-limit superior decoding performance through iterative processes. Furthermore, the decoder uses a "turbo" feedback and the method has been generalized to "Turbo-Principle" [5].

For the turbo decoding, there should be a decoding threshold (B position in Fig. 1(a)), where the decoder must spend infinite iterations (bold-dashed line) to overcome the seriously noisy channel. Considering of the cost of the hardware implementation, several schemes are usually employed in practical VLSI designs: 1) Fixed iteration chart (Fig. 1(b)) - The decoder will perform decoding with the max iteration under any SNR. The designer usually adopts the fixed decoding iteration number (A position in Fig. 1(b)) according to the requirement of some specification, such as the bold-solid line of Fig. 1(b). 2) Conventional stopping chart (Fig. 1(c)) - The decoder will stop decoding (early termination) in high-SNR situation where the decoded bits are high reliable. In the noiseless (high-SNR) environment, the decoder can easily complete the decoding. Instead of fixed-iteration decoding, the decoder can stop operating in early state.

In recent literatures [6]-[13], some stopping criteria for turbo decoders have been presented, and they can be categorized into two classes: One class is based on soft-bit decisions, such as Cross-Entropy (CE) [6], Mean-Estimation (ME) [7] Updated Threshold [7], and A-priori LLR Measurement [8]. The other class is based on hard-bit decisions, such as Sign- Change-Ratio (SCR) [9], Hard-Decision-Aided (HDA) [9], Sign- DifferenceRatio (SDR) [10], and Improved-HDA (IHDA) [11]. In addition, some methods are based on extra checking policies, such as Cyclic-Redundancy- Check (CRC) [12] and Valid- Code-Word check (VCW) [13].

However, they only focus on the solvable decoding, that is, the decoder always can recovery correct information bits even with serious noise interference. Obviously, unlimited decoding ability is impossible. The decoding limitation has been discussed in the literatures, where the SNR [14][15] or mutual information [15] measurements are used to build the extrinsic information transform (EXIT) chart and then to observe the convergence of iteratively decoding. In this paper, we propose another measurement of using cross correlation and discussed the relation between them.

For an iteratively computing design, there is usually an operating limit, in which any further iteration results in very little improvement. Therefore, it is important to derive an effective criterion to stop the iterative process, so as to prevent from unnecessary computations and decoding delay. In this paper, we proposed a new ET scheme (Fig. 1(d)) - The decoder will stop in either high-SNR situation where the decoded bits are high reliable, or in low-SNR situation where the decoder no longer has the capability to decode the incoming data.

The proposed ET scheme is based on the (infiniteiteration) decoding threshold, obtained by the EXIT chart. At the same time, the receiver can send an automatic-repeat-request (ARQ) message to the transmitter in noise-seriously-coupling situation. As a result, our proposed ET scheme can either reduce the computational complexity or power consumptions in implementing the iterative decoding in DSP processors or VLSIs in mobile handsets, and the decoder can be 
terminated earlier while achieving the system specification.

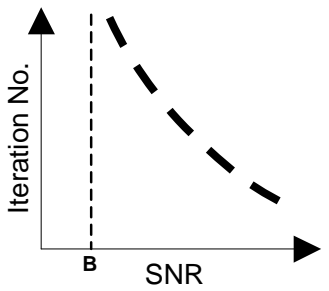

(a)

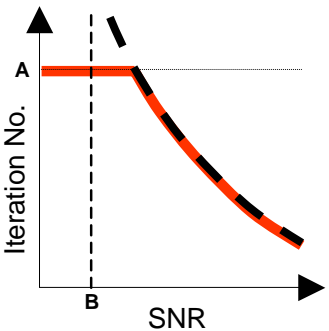

(c)

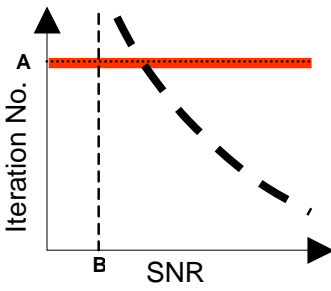

(b)

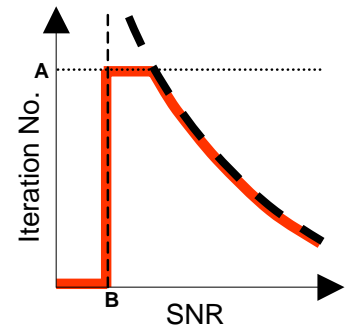

(d)
Fig. 1. Iteration number vs. SNR: (a) Iteration nature.

(b) Fixed-iteration chart. (c) Conventional-stopping chart (d) Proposed ET scheme.

\section{EXTRINSIC INFORMATION TRANSFER CHART}

The conventional turbo decoder is composed of two component decoders. Based on iteratively decoding, the input a-priori LLR of one component decoder comes from the output extrinsic LLR of the other component decoder [1][2]. The literatures [14][15] show that the EXIT chart of single component decoder can characterize the convergence of the turbo decoder. Fig. 2 shows the EXIT flow that using the Gaussianapproximated LLR to measure the relation between the input and the output of the component decoder.

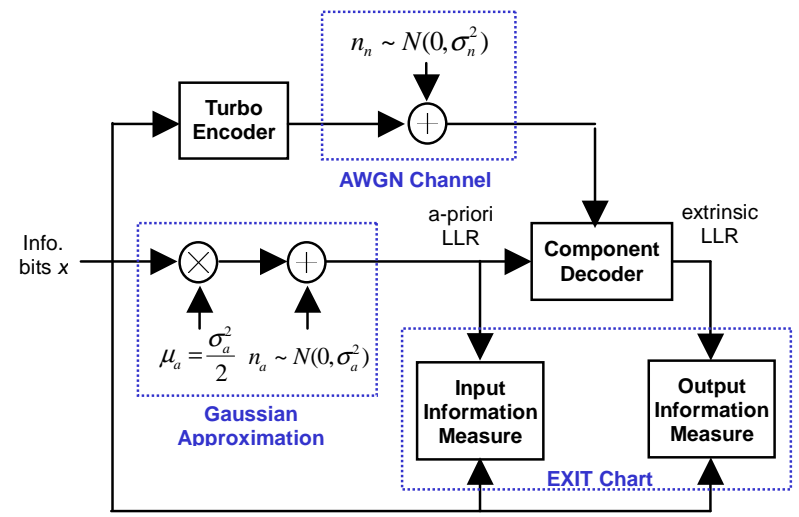

Fig. 2. Extrinsic information transfer (EXIT) diagrams [15].

\subsection{Gaussian Approximation Log-Likelihood-Ratio}

For the binary symmetric signal $x$ passing through AWGN channel, the received discrete-time signal is

$$
z=x+n
$$

where $n$ is zero mean and $\sigma_{n}^{2}$ variance. The conditional probability density function of $z$ writes as

$$
\operatorname{Pr}(z \mid x=\mu)=\frac{1}{\sqrt{2 \pi} \sigma_{n}} \exp \left(\frac{-(z-\mu)^{2}}{2 \sigma_{n}^{2}}\right) .
$$

The binary random variable $x$ denotes the transmitted bits with relations $\mu \in\{ \pm 1\}$. The LLR-values $Z$ are calculated as

$$
Z \equiv \log \frac{\operatorname{Pr}(z \mid x=+1)}{\operatorname{Pr}(z \mid x=-1)}=\frac{2}{\sigma_{n}^{2}} z=\frac{2}{\sigma_{n}^{2}}(x+n),
$$

which can be simplified as

$$
Z \equiv \mu_{Z} x+n_{Z}
$$

with

$$
\mu_{Z}=\frac{\sigma_{Z}^{2}}{2} \text {. }
$$

For soft in/soft out decoding with BCJR-algorithm, the priori information $L_{a}$ can be modeled by applying an independent Gaussian random variable $n_{a}$ with variance $\sigma_{a}^{2}$ and mean zero corresponding to the known transmitted systematic bits $x$ [14].

$$
L_{a} \equiv \mu_{a} x+n_{a}
$$

where

$$
\mu_{a}=\frac{\sigma_{a}^{2}}{2}
$$

The probability density function is

$$
p\left(L_{a} \mid x=\mu\right)=\frac{1}{\sqrt{2 \pi} \sigma_{a}} \exp \left(-\frac{\left(L_{a}-\frac{\sigma_{a}^{2}}{2} \mu\right)^{2}}{2 \sigma_{a}^{2}}\right)
$$

\subsection{SNR Measurement}

To measure the information, a signal-to-noise ratio [14] for the random variable $L_{a}$ can be defined as

$$
S\left(L_{a}\right) \equiv \frac{E\left[L_{a}\right]^{2}}{\operatorname{Var}\left[L_{a}\right]}
$$

\subsection{Mutual Information Measurement}

Another way to measure the information between transmitted systematic bits $X$ and $L_{a}$ is used [15].

$$
I\left(X ; L_{a}\right) \equiv E\left[\log _{2} \frac{p\left(L_{a} \mid X\right)}{P\left(L_{a}\right)}\right] .
$$

Then, the mutual information can be obtained by the histogram measurement of $p\left(L_{a} \mid X\right)$. Furthermore, with Eq. (8),

$$
\begin{aligned}
& \frac{p\left(L_{a} \mid X=+1\right)}{P\left(L_{a}\right)}=\frac{2}{1+e^{-L_{a}}}, \\
& \frac{p\left(L_{a} \mid X=-1\right)}{P\left(L_{a}\right)}=\frac{2}{1+e^{+L_{a}}}
\end{aligned}
$$


Then, Eq. (10) becomes

$$
I\left(X ; L_{a}\right)=E\left[\log _{2} \frac{2}{1+e^{-X L_{a}}}\right] .
$$

Thus, the mutual information can be obtained by the time average.

\subsection{Cross Correlation Measurement}

For the iterative decoding, the priori probability $p(X)$ is updated by the extrinsic information.

$$
\begin{aligned}
& p\left(X=+1 \mid L_{a}\right)=\frac{e^{L_{a}}}{1+e^{L_{a}}}, \\
& p\left(X=-1 \mid L_{a}\right)=\frac{1}{1+e^{L_{a}}} .
\end{aligned}
$$

Thus, we proposed another information measurement. Based on the estimation of systematic bits $\hat{X}$ that $\hat{X}=(+1) p(X=+1 \mid L a)+(-1) p(X=-1 \mid L a)$,

the information measurement can be computed by the cross correlation between the estimated systematic bits $\hat{X}$ and the known ones $X$.

$$
C(X ; \hat{X}) \equiv E[X \hat{X}]=E\left[X \frac{e^{L a}-1}{1+e^{L a}}\right]
$$

Moreover, the Eq. (14) can be expressed as

$$
\hat{X}=\frac{p(L a \mid X=+1)-p(L a \mid X=-1)}{2 p(L a)} .
$$

Thus, the cross-correlation can be formularized in another form by

$$
C(X ; \hat{X})=E\left[\frac{p\left(L_{a} \mid X\right)}{p\left(L_{a}\right)}-1\right] .
$$

Compared with the mutual information measurement, the cross correlation also can be computed by the histogram measurement in Eq. (17) and time average in Eq. (15). The simulations for the variance $\sigma_{a}^{2}$ are shown in Fig. 3.

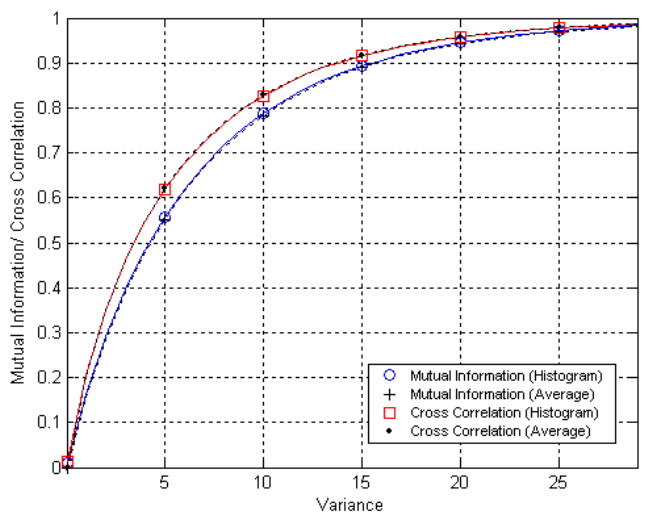

Fig. 3. Information measurement vs. variance $\sigma_{a}^{2}$.

It shows the result of the histogram measurement is the same with the one of the time average, and the variation of the cross correlation is similar to the one of the mutual information. Both measurements are bounded between zero and one.

\subsection{EXIT Chart}

To observe the convergence of iteratively decoding, the transfer functions of the component decoder, which are the information measurements of input and output, are plotted twice. The axes of one decoder are swapped, and thus the output of one decoder is the input of the other. The exchange of extrinsic information can be visualized as a decoding trajectory. For the noisy channel, the decoder must spend more iteration to pass the narrow tunnel. Consequently, when the connection of both curves occurs, the decoding threshold can be obtained since the decoding trajectory cannot pass.

\section{PROPOSED EARLY TERMINATION SCHEMES}

\subsection{Proposed ET Schemes}

Except the early termination due to the successful decoding in high SNR channel, the decoder also needs to stop operating in low SNR channel since the decoded frames cannot satisfy the system specification. For the above EXIT discussion, the decoder has the decoding limitation. Therefore our proposed ET scheme is that the decoder can stop operating behind the (infinite-iteration) decoding threshold. Fig. 4 shows the design flow of our approaches, which are partitioned into off-line and on-line computing blocks. The off-line blocks are to obtain the ET thresholds according to the EXIT charts or specifications. The on-line blocks are to operate real time with the decoder.

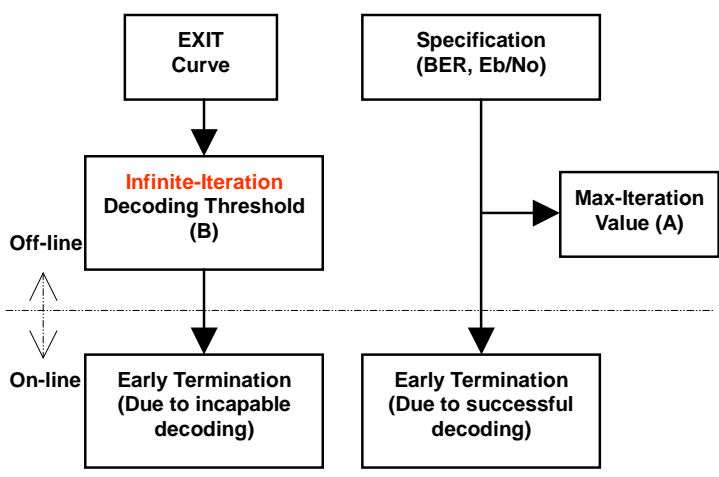

Fig. 4. The proposed early termination (ET) scheme.

\subsection{Characteristics of ET Indicators}

For the above ET discussion, most of all use the absolute value or sign of the log-likelihood-ratio to be the ET indicators. Fig. 5 shows the ET indicator variation for the SNR. In the high-SNR situation, all indicators can work well since they vary monotonically. However, in the low-SNR situation, the indicators of SCR, HDA, and IHDA are near quadratic variation, which interferes the judgment of the ET function. As a result, the SCR, HDA, and IHDA approaches are not suitable in the low-SNR situation. 


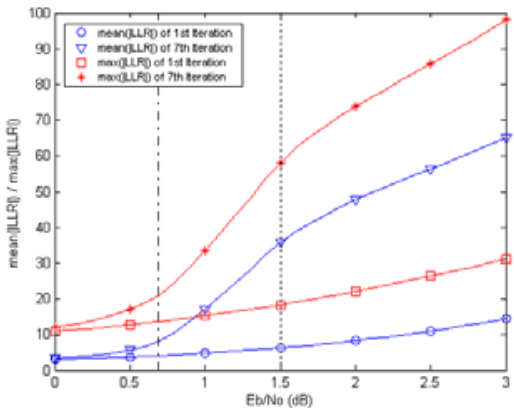

(a)

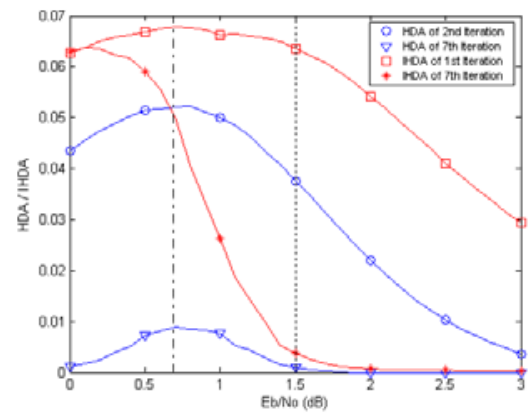

(b)

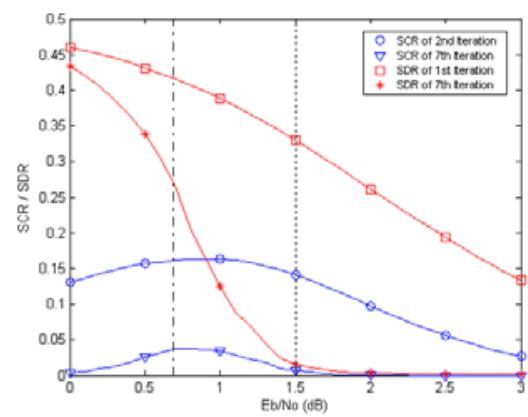

(c)

Fig. 5. ET indicators vs. Eb/No: (a) mean and maximal value of absolute LLR. (b) HDA and IHDA. (c) SCR and SDR.

\subsection{Measurement of Reliability (MOR)}

In the turbo decoding algorithm [1][2], the magnitude of LLR itself is an index that indicates the reliable degree of decoded information bit. Thus, we define the magnitude of LLR $\left|L_{2}^{(i)}\left(\hat{u}_{k}\right)\right|$ as reliability $R_{k}$ by

$$
R_{k}=\left|L_{2}^{(i)}\left(\hat{u}_{k}\right)\right| .
$$

Clearly, the decoding is complete and can be terminated if the reliability of all decoded bits is greater than some threshold value, $T h_{1}$.

$$
\min _{k \in\{1,2, \ldots, N\}}\left[R_{k}\right]>T h_{1} .
$$

Besides, if the noise is so larger that the decoder can do nothing, that is, the reliability of all decoded bits is smaller than some threshold value, $T h_{2}$.

$$
\max _{k \in\{1,2, \ldots, N\}}\left[R_{k}\right]<T h_{2} \text {. }
$$

The decoder can stop decoding and send the automatic-repeat-request (ARQ) message to the transmitter.

\subsection{Frame Testing and Stage Testing}

Both Eqs. (19) and (20) show that the testing condition is based on the overall frame. Thus, the approach needs $2 N$ comparison operations per iteration.

By logical relationship, the condition of stopping decoding as the reliabilities of all decoded bits are greater (smaller) than the threshold, can be changed by the condition of continuing decoding as the reliability of any decoded bit is smaller (greater) than the threshold. If the reliability of any decoded bit matches the condition, the one of others need not to be tested again and the comparison can be switched off. As a result, the comparison operations can be reduced.

\subsection{Modification on Existing Methods}

Although there are several approaches discussed above, most of all focus on stopping in high SNR. If the noise is serious, the decoder usually performs with maximum iterations, even out of the decoding ability. According to our stopping concepts, some approaches can be modified to stop in low-SNR situation, such as SCR, SDR, and ME methods.

\section{A. $\quad$ Modified-ME (M-ME)}

In addition to the condition of ME in [7], we add anther condition of stopping in low SNR that

$$
M_{|L|}<T h^{\prime},
$$

where $T h^{\prime}$ is a pre-defined threshold that the mean of absolute LLR can not be greater than in low SNR.

\section{B. Modified-SCR (M-SCR)}

For low SNR, it is shown that the sign of LLR changes rapidly. Therefore, in addition to the condition of SCR in [9]), we add anther condition of stopping in low SNR that

$$
C(i)>T h,
$$

where $T h$ is a pre-defined threshold that the frequency of sign change is greater than in low SNR.

\section{C. $\quad$ Modified-SDR (M-SDR)}

Similar to M-SCR, in addition to the condition of SDR in [10]), we add anther condition of stopping in low SNR that

$$
D(i)>T h,
$$

where $T h$ is a pre-defined threshold that the frequency of sign change is greater than in low SNR.

\section{SIMULATIONS AND COMPARISONS}

We perform simulations for 4-state Turbo codes, which generator polynomials are 7 and 5. The Turbo-codes are obtained for hundreds of frames, where length $N$ is 1000 , and an overall rate $R$ is $1 / 2$. The reductions of decoding iterations using different stopping criteria for the iterative Maximum A-posteriori Probability (MAP) [3][4] decoding scheme are considered. Moreover the requirement of the system specification is assumed that 
the decoder must satisfy $10^{-3}$ bit error rate (BER) above $1.5 \mathrm{~dB}$ AWGN channel.

\subsection{Decoding Threshold}

To find the decoding threshold, three information measurements are adapted in the AWGN channel from $0.6 \mathrm{~dB}$ to $1.2 \mathrm{~dB}$ with step size $0.1 \mathrm{~dB}$. Fig. 6(a) show that the transfer curves of SNR measurement close to diagonal line as the channel becomes noisier. Then Fig. 6(a) are rotated 45 degrees clockwise and plotted in Fig. 6(b). Mutual information and cross correlation measurements can also be performed with the same steps. Fig. 7 shows the curves composed of the lowest points of the transfer curves in Fig. 6(b). As a result, the decoding thresholds, obtained from the cross with the zero horizontal line, are $0.69 \mathrm{~dB}$ by the mutual information measurement, $0.76 \mathrm{~dB}$ by the cross correlation measurement, and $0.80 \mathrm{~dB}$ by the SNR measurement. For the following simulations, we adopt the optimistic decoding threshold $0.69 \mathrm{~dB}$ to avoid missing the decodable patterns.

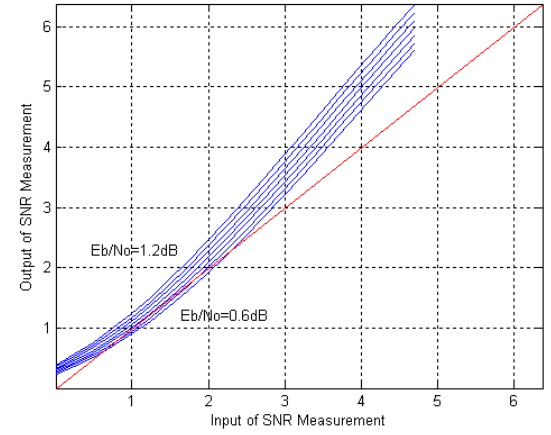

(a)

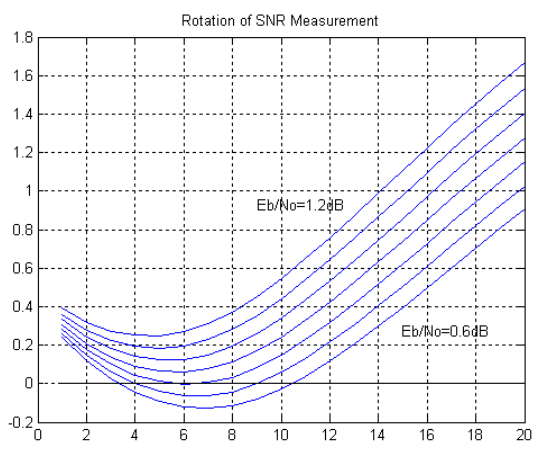

(b)

Fig. 6. Simulations of decoding trajectory.

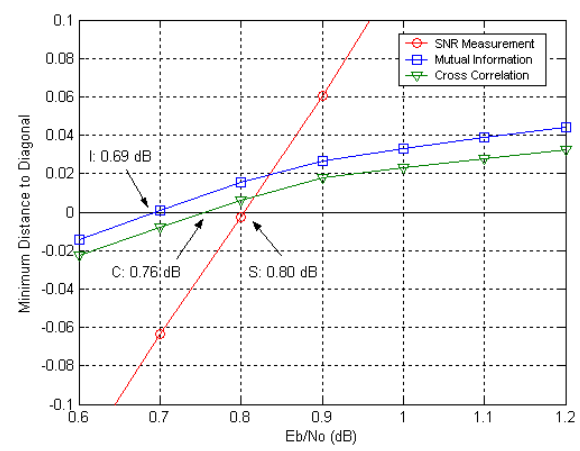

Fig. 7. Simulations of decoding threshold.

\subsection{Simulations for GENIE-Aided Benchmark}

For the specification of $10^{-3} \mathrm{BER}$ at $1.5 \mathrm{~dB}$, the required iterations of the decoder is seven, given by the simulation. Before simulating the above stopping approaches, it needs a GENIE-aided benchmark to be the performance boundary. It can be found that the performances of last iterations are so close that further iterations increase little improvement; besides, with increasing iterations, the BER has no improvement in low SNR. The GENIE curve (maximal iteration $=7$ ) is used in previous stopping approaches, and its BER performance is almost the same as the one of the fixed-7-iteration decoding. For the proposed ET scheme, there is another GENIE curve, below the infiniteiteration decoding threshold $0.69 \mathrm{~dB}$, just runs an iteration to obtain the LLR for stopping-criterion testing. The BER performance curve of the ET scheme is composed of the one-iteration and seven-iteration decoding, separated at $0.69 \mathrm{~dB}$.

\subsection{Simulations for Different Stopping Criteria}

Then, with the conventional GENIE curve, the simulations for several approaches discussed above are performed. Fig. 8(a) illustrates the simulations of iterations vs. Eb/No for SCR, SDR, HDA, IHDA, and $\mathrm{ME}$ approaches. It can be found that in high SNR, the sequence about the quantity of reduced iterations is as follows:

$$
\mathrm{ME}, \mathrm{SDR}>\mathrm{SCR}>\mathrm{HAD}>\mathrm{IHDA} \text {. }
$$

However, they perform with maximal iterations in low SNR. Because of the non-monotonic variation of the SCR indicator in Fig. 5(c), the SCR based on threshold comparing is ineffective in low-SNR situation in Fig. 10(a). Last the BER performance simulations are performed in Fig. 8(b). Besides, we also add the simulations of conventional GENIE (maximal iteration = 7) and fixed-7 iteration decoding as the benchmark. All of them can satisfy the specification.

Then, Fig. 9(a) illustrates the simulations for MOR, M-SCR, M-SDR, and M-ME approaches using the proposed ET scheme. Because of the non-monotonic variation of the SCR indicator in Fig. 5(c), the low-SNR and high-SNR stopping criteria of M-SCR will interfere with each other and be failed. Note that the pre-simulated ET thresholds must satisfy that the iterations at $0.69 \mathrm{~dB}$ must exceed the ones of GENIE (maximal iteration $=7$ ) in Fig. 9(a), which keeps the decoder normally working over the infinite-iteration decoding threshold. It can be found that in low SNR, the sequence about the quantity of reduced iterations is as follows:

$$
\mathrm{M}-\mathrm{ME}>\mathrm{MOR}>\mathrm{M}-\mathrm{SDR} \text {. }
$$

Last, the performance of them is close either in low-SNR or high-SNR situations in Fig. 9(b), which also satisfy the specification. 


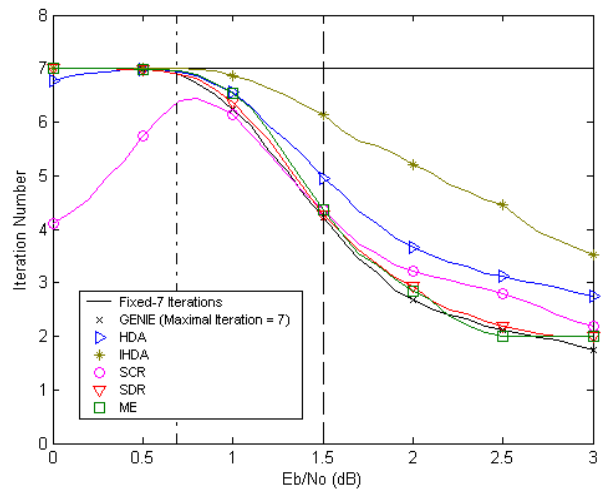

(a)

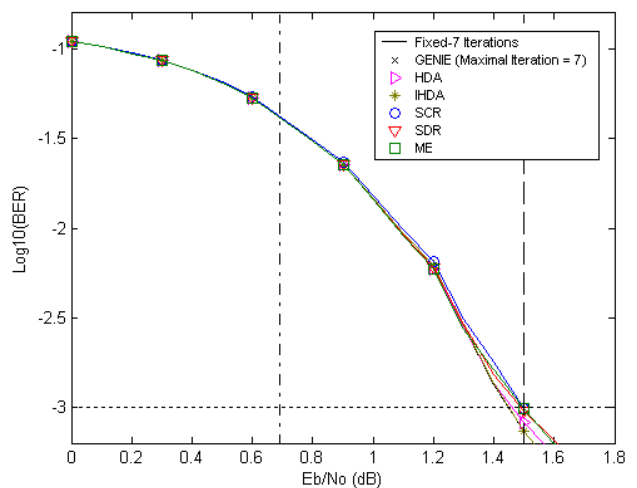

(b)

Fig. 8. Conventional ET Strategies: (a) Simulations for Iteration vs. Eb/No. (b) Simulations for BER vs. Eb/No.

\section{v. CONCLUSIONS}

This paper discusses the iterative decoding threshold. Using the decoding threshold, a new ET scheme is proposed. The iterative decoding can stop in either high-SNR situation that the decoded bits are high reliable, or in low-SNR situation that the decoder already has no ability to decode. By the simulations, the reduced iterations due to early termination and the saved comparison operations due to stage testing still satisfy the system specification.

\section{REFERENCES}

[1] C. Berrou, A. Glavieux, and P. Thitimajshima, "Near Shannon limit error-correcting coding and decoding: Turbo codes," in Proc. ICC, pp. 1064-1070, May 1993.

[2] C. Berrou and A. Glavieux, "Near optimum error correcting coding and decoding. Turbo-codes, "IEEE Trans. Commun., vol. 44, no. 10, pp. 1261-1271, 1996.

[3] L. Bahl, J, Cocke, F. Jelinck, and J. Raviv, "Optimal decoding of linear codes for minimizing symbol error rate," IEEE Trans. Inform. Theory, vol. IT-20, pp. 284-287, March 1974.

[4] P. Robertson, E. Villebrun, and P. Hoeher, "A comparison of optimal and sub-optimal MAP decoding algorithms operating in the log domain," in Proc. Int. Conf. Communications, June 1995, pp. 1009-1013.

[5] J. Hagenauer, "The turbo principle: Tutorial Introduction and state of the art," in Proc. Int. Symp. Turbo Codes and Related Topics, Brest, France, 1997, pp. 1-11.

[6] J. Hagenauer, E. Offer, and L. Papke, "Iterative decoding of binary block and convolutional codes," IEEE Trans. Inform. Theory, vol. 42, pp. 429-445, Mar. 1996.

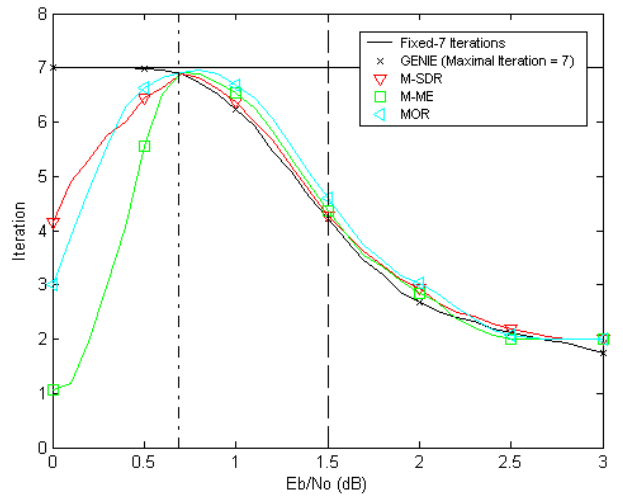

(a)

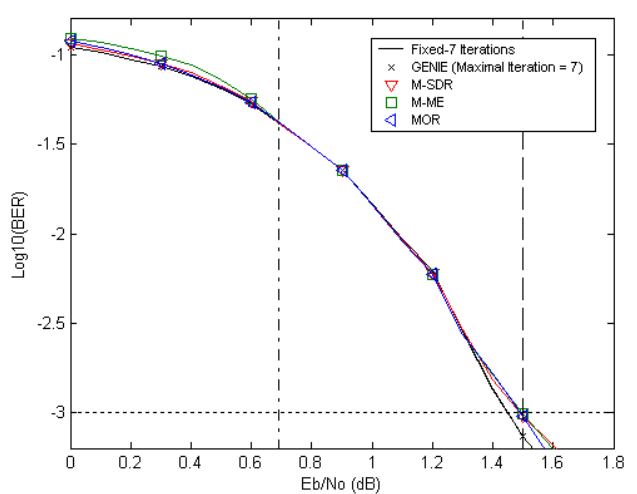

(b)

Fig. 9. Proposed ET scheme: (a) Simulations for Iteration vs. Eb/No. (b) Simulations for BER vs. Eb/No.

[7] F. Zhai and I. Fair, "New error detection techniques and stopping criteria for turbo decoding," in Proc. 2000 Canadian Conference on Electrical and Computer Engineering, vol. 1, pp. 58-62, Mar. 2000. N. Y. Yu, M. G. Kim, Y. S. Kim, and S. U. Chung, "Efficient stopping criterion for iterative decoding of turbo codes," IEE Electronic Letters, vol. 39, pp. 73-75, January 2003.

[8] D. Bokolamulla and T. Aulin, "A new stopping criterion for iterative decoding," IEEE Commun. Society, pp. 538-541. 2004.

[9] R. Y. Shao, S. Lin, and M. P. C. Fossorier, "Two simple stopping criteria for turbo decoding," IEEE Trans. Commun., vol. 47, pp. 1117-1120, Aug. 1999.

[10] Y. Wu, D. Woerner, and J. Ebel, "A simple stopping criteria for turbo decoding," IEEE Commun. Letters, vol. 4, pp. 258-260, Aug. 2000.

[11] T. M. N. Ngatched and F. Takawira, "Simple stopping criterion for turbo decoding," IEE Electronic Letters, vol. 37, pp. 1350-1351, Oct. 2001.

[12] A. Shibutani, H. Suda, and F. Adachi, "Reducing average number of turbo decoding iterations," IEE Electronic Letters, vol. 35, pp. 701-702, Apr. 1999.

[13] J. Heo, K. Chung, and K. M. Chugg, "Simple stopping criterion for min-sum iterative decoding algorithm," IEE Electronic Letters, vol. 37, pp. 1530-1531, December 2001.

[14] H. E. Gamal and A. R. Hammons, "Analyzing the Turbo Decoder Using the Gaussian Approximation," IEEE Trans. Inform. Theory, Vol. 47, No. 2, pp. 671-686, Feb. 2001.

[15] S. T. Brink, "Convergence Behavior of Iteratively Decoded Parallel Concatenated Codes," IEEE Trans. Commun., Vol. 49, No. 10, pp. 1727-1737, Oct. 2001. 